\title{
BRIDGING THE GAP - FROM PROCESS RELATED DOCUMENTATION TO AN INTEGRATED PROCESS AND APPLICATION KNOWLEDGE MANAGEMENT IN MICRO SYSTEMS TECHNOLOGY
}

\author{
Markus Dickerhof, Anna Parusel \\ Forschungszentrum Karlsruhe \\ Phone: $+49724782-5754$ \\ Fax: $+49724782-5786$ \\ Email: dickerhof@iai.fzk.de
}

\begin{abstract}
Knowledge about the relations between process steps or dependencies of materials, technologies, designs, tools and machines is mostly stored in the product developers mind. The same effect takes place when one talks about product related knowledge and the referring technological requirements It is lost if the person leaves the company. The following paper describes a knowledge management approach for a product independent description of Micro System processes and presents a software prototype for decision support in early product development phases.
\end{abstract}

Keywords process knowledge management, design for X, decision support systems

\section{Knowledge Management in MST Industry}

Planning development and production today means proceeding along economic and technical parameters, derived from product requirements and customer oriented business processes. During the last few years much effort has been made to push "Knowledge Management" in industry. At the least, the result often represented no more than a new type of document management with additional "meta" information on top to support the requirements of a company's quality management. Documentation of technological capabilities in Microsystems Technology (MST) often leads to a non-reusable, product specific documentation of a production design according to the quality management guidelines. Hence, a lot of information about the subjacent fabrication know-how - or relations between application requirements, physical effects, technological constraints and costs, - is so far not documented, or documented in an insufficient manner. Insufficient in this context means irretrievable or only customer project related so that it cannot be reused for new projects in terms of Nonaka and Takeuchi's Seci model [01]. Knowledge about

Please use the following format when citing this chapter

Dickerhof, M., Parusel, A., 2008, in IFIP International Federation for Information Processing, Volume 260, MicroAssembly Technologies and Applications, eds. Ratchev, S., Koelemeijer, S., (Boston: Springer), pp. $109-119$. 
the relations between process steps or dependencies of materials, technologies, designs, tools and machines is mostly stored in the product developers mind. The same thing occurs with regard to the relation of product requirements and referring technological capabilities. It is lost if the employee leaves the company. Also the "neutral" transfer of information or knowledge about risks from one project to another can even be difficult for a person, because of the complexity that has to be considered. A fortiori know-how transfer is extremely expensive and time intensive for a company, and is therefore worth handling in a well-structured economic manner. The following paper describes an approach allowing us to overcome some of the limitations in present product development through the use of a decision support system based on a MST-Ontology.

\subsection{Knowledge and knowledge management in the industrial production context}

The terms "knowledge" and "knowledge management" are frequently used in information technology in many respects. Their scope of use ranges from the mapping of simple structural knowledge to the representation of knowledge by means of semantic networks or methods of artificial intelligence.

Application of these approaches is certainly feasible in an enterprise and useful in various fields where enterprise knowledge is generated. The present contribution, however, will focus on product development or fabrication knowledge and in particular on the necessary process know-how especially in the MST context (Fig 1). As MST is an interdisciplinary field of technology, special attention needs to be drawn on influencing factors, coming from market / application requirements and related physical principles.

The percentage of work done in that direction demonstrates the early state of industrial implementation, especially when considering that the numbers do not represent the availability of knowledge within the enterprise.

In industry, work related to the storage of knowledge has advanced to different levels. Large enterprises with complex fabrication processes undertake considerable efforts in acquiring knowledge and integrating it in a "learning" enterprise (which also covers the "supply chain", mostly according to a specific product development and production process).

As far as small and medium-sized enterprises are concerned, this aspect so far has met with less attention, as their in-house process chains are less complex. With increasing product complexity and virtualisation of production chains, the necessity of joining development and production capabilities is essential for small enterprises to be able to produce complex products.

As with large companies, the key for success in such a company's overlapping development is also the process-overlapping knowledge management. This particularly applies to micro systems technology, where description standards are rare. 

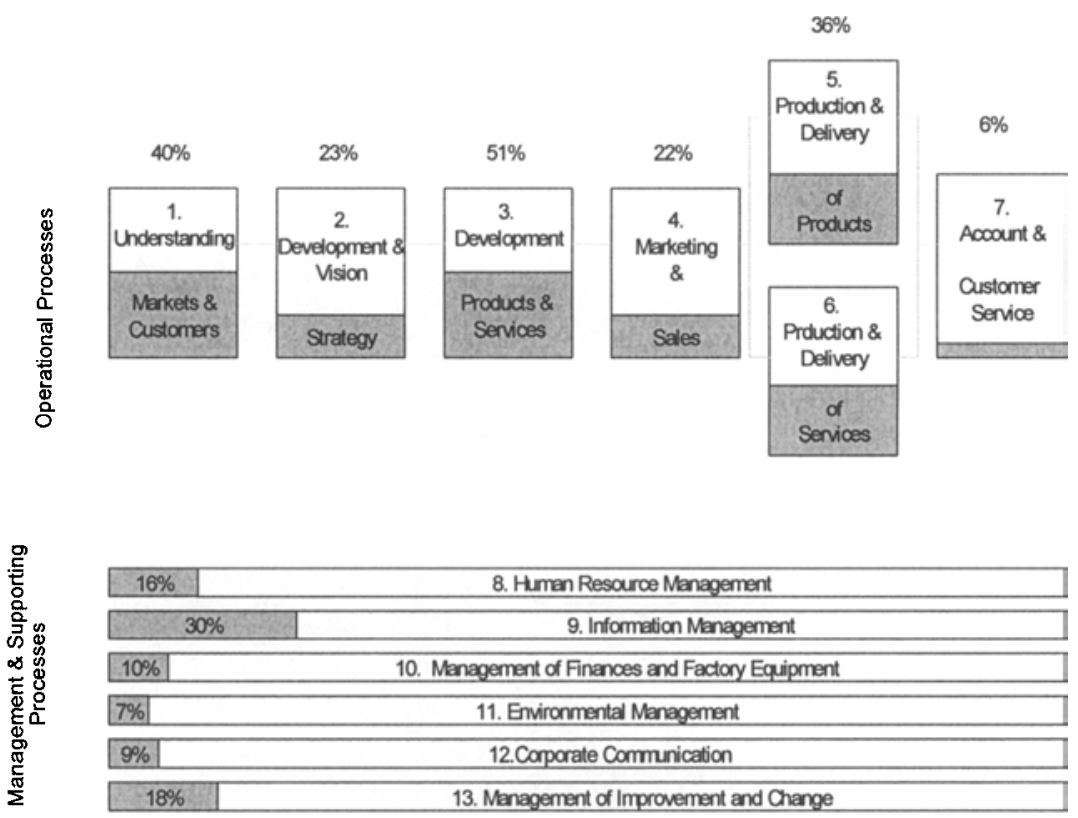

Fig. 1. Knowledge Management in an Enterprise [02]

A number of software systems today claim to map enterprise knowledge. However, no system is known (especially for non silicon MST) which processes the data collected during the product and production development process in such a way that the "evolution" of the process parameters can be assessed or patterns and regulations (design rules) can be derived from the process-overlapping connections of the interdisciplinary partial areas of micro systems technology in analogy to the rules defined in silicon micro systems technology.

\subsection{Knowledge management in the development and production phase}

The basic idea of the ProWiDa concept is to support product developers with the identification of the best fitting technological approach for a given product requirement (in this specific context, the term technology shall be considered in a wider context: technology, AND manufacturing). It is based on the assumption that for reusability of information - collected during a product development process - the acquisition of MST development and fabrication phase should proceed in a order -, application- and product independent manner.

As micro systems are typically integrated as parts, components or subsystems into macro systems, one has to divide between parameters that are of relevance for the product and technological parameters that are dedicated to a technology itself. 
The former mostly cover economic constraints requirements or describe capabilities of a technology, e.g. related to the physical effects to fulfill the task given (e.g. surface roughness as a factor of influence for optical properties). The latter allows the user to identify the technology itself (material, technology, design, equipment, tools, etc.)

Anyway, such a methodology for the description of relations between technological parameters and application requirements can be seen as a tool towards "Design for $X$ " approach. To reach the goal of a reusability of information it should be based on an identification of application-independent parameters as a basis for reusability of development and industrialisation results gathered in another context.

Industrial activities in this field are under discussion in white papers and other documents [03]. Scientific activities such as the pretzel model present models for the Silicon-MST field [04]. Sadly the approaches are not easily transferable though the increased complexity coming from the huge flexibility in materials [05]. First activities on a coordinative level into this direction are currently started under the roadmapping activities of the EU Coordination Action Microsapient, attempting to link application requirements with technological capabilities on a very high level. Figure 2 gives a schematic overview on this relation.

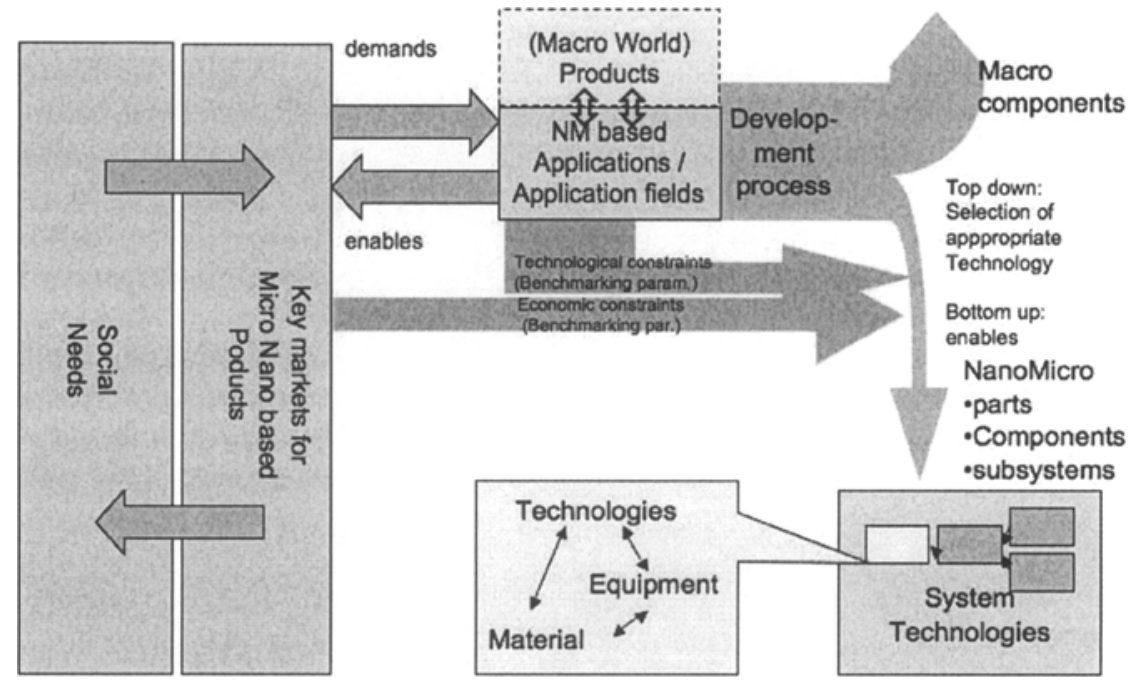

Fig. 2. Bottom up - Top down approach of Product development in MST

In the following chapter the focus will be on the description of a methodology for technological aspects in the above mentioned manner (see also lower right section of Figure 2). 


\subsection{Knowledge management of technical aspects in MST}

Today the classification parameters of MST technologies are often mixed, consisting of production related parameters, design rules and sometimes (estimation of expected) product properties:

- Process Parameters describe typical technical factors that can be achieved in a reliable manner (width, length, material parameters, milling tool size, feed, milling velocity, etc)

- Design Rules (or product parameters) describe the resulting capabilities, e.g. resulting minimum grooves, surface roughness, which again correlate with application specific requirements coming from physical effects often utilised in Sensor or actuator applications.

- As it is not possible to directly match technological parameters with application requirements in a product independent manner as long as these are not defined with a specific approach in mind, we defined an additional "physical effects" layer, allowing for the linkage of process capabilities with application requirements via an application independent description of "physical properties".

An analysis of the most influencing production factor resulted in a set of up to six factors of influence, in ProWiDa called aspects, for the characterisation of process (step) related parameters:

- Materials (substrates and layers)

- Procedure

- Geometry

- Machines

- Tools

A process step as the smallest modelling element consists of $n$-tuple of parameters sets attached to the aspects mentioned before. Additional parameter sets can be attached to the competence itself (e.g. product properties related to a specific combination of material, design and technology). These combinations (competences) can be additionally attached to a specific group or company, if the knowledge shall be shared as a knowledge map in a distributed environment. The approach describing the prototype which has been realised so far is outlined in the next chapter. A schematic overview is presented in Figure 3.

- Material, procedure and geometry are almost sufficient for a clear definition of parameter sets that can be approximately compared with the Design Rules, we already know out of the silicon MST. These parameter sets are of relevance for a design engineer or the marketing department to check out the feasibility of a customer' request.

- The remaining aspects are important for the clear and reproducible definition of fabrication parameters you need at the shop floor level. Remark: Not every aspect needs to be of relevance for a proper description of a competence. 


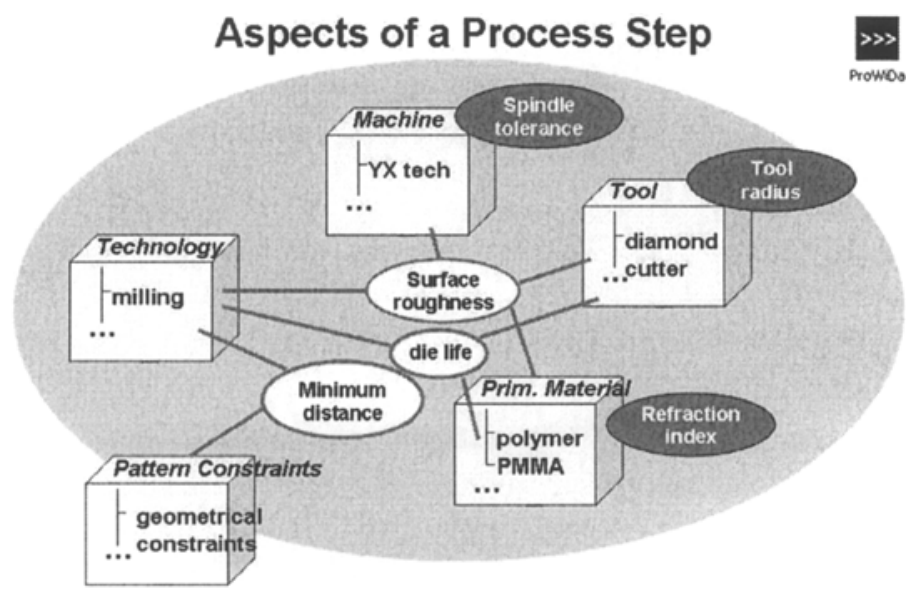

Fig. 3. Technology-oriented "Aspects" of a process step

\subsection{Knowledge about relations and constraints between process steps.}

State of the art process documentation is typically realised along the requirements of a quality management system, which usually just describes the relevant business process and - as a subset - the product related production chain. We aimed to find a way to show the relations between the process steps in a more flexible and transferable manner, which also allows the user to store information about "Meta" parameters. These Meta-parameters are additional properties or differing properties resulting out of the combination of single process steps to a process sequence or process chain.

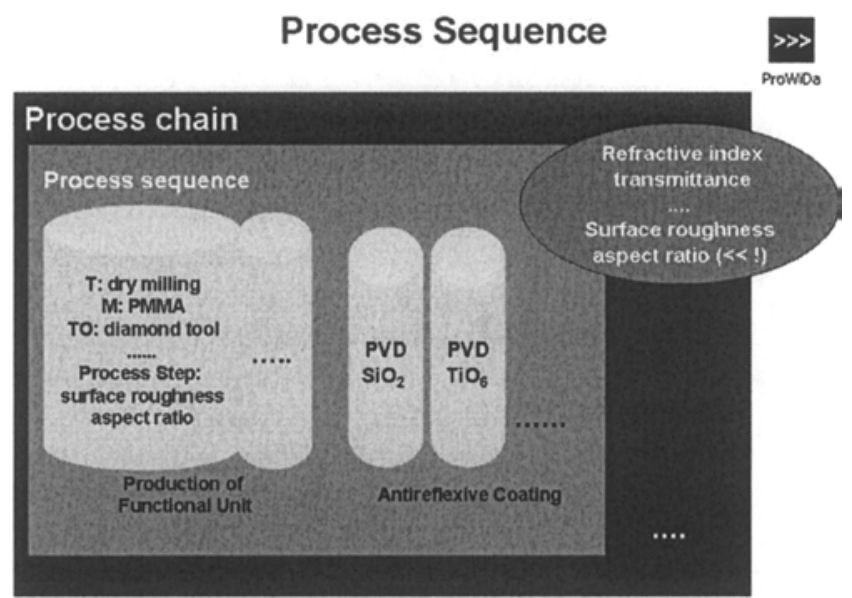

Fig 4. Example of a production process for an optical surface using generic modelling elements 
Most work in this field suffers from a lack of real world process parameters which are imperative to serve as a basis for the analysis of processes and process relations, e.g. with AI methods.

\section{The ProWiDa System}

For acquisition and order-independent filing of process parameters (esp. during the development phase) a database approach with a web based user interface for the augmentation of the feasibility studies and product development cycle in a distributed SME network was developed and successfully tested [06]. The system today consists of five modules:

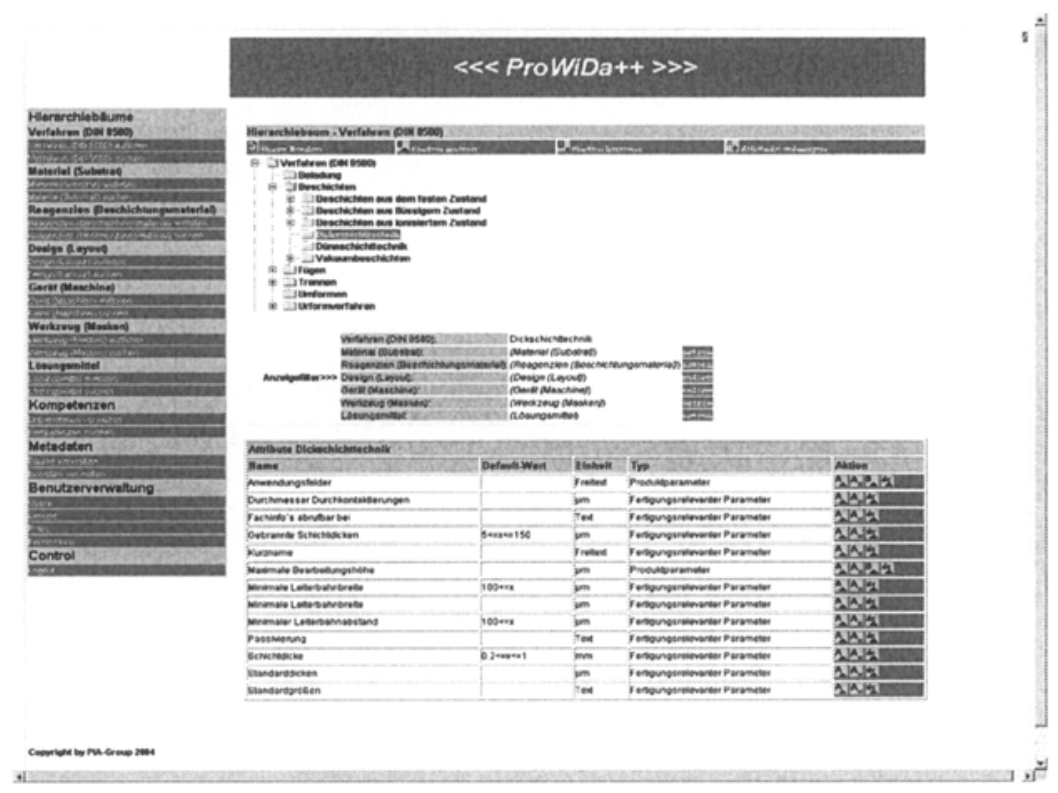

Fig 5. Aspects and parameter sets attached to a competence

\subsection{Parameter classification module}

The parameter module (Fig. 5) is one of the core elements of the ProWiDa System. Like many database driven onthologies it is based on generalisation hierarchies for the description and structuring of the major influencing aspects of a technology. These hierarchies can be compared with the trees structure of the file system on a PC. The place where one branch is attached to the next is called a node. At each node level information can be stored, inherited or generalised to the next levels 
above or below. This allows for the definition of template structures and parameters that can be e.g. refined on the next level of detailing. In this way, technologies, materials and other aspect can be structured in a standardised manner.

In ProWiDa - instead of information elements or files - process attributes and values are assigned to each node and leaf of the hierarchy tree. Each tree represents an aspect as defined in Chapter 3. Summation of parameter sets out the different hierarchy trees then yields a list of parameters for the "competence" on the lowest levels of the tree.

The values of specific parameter sets attached to the process step in a specific sequence describe an order related process chain. If one only would look at a specific combination of these parameter sets, there would be at first sight no difference to the specification of a work list being stored in a database system.

Filters allow for a retrieval of the competences e.g. one can mask out the material parameter dependency. This is useful as an indication for a first feasibility estimation if there exists no parameter set including the specified material.

The parameter module allows also for the integration of competence- or process sequence specific additional information, documentation (e.g. pictures, documents, hyperlinks).

Customized features like integration of related FMEA-templates and links to work list have also been realized.

\subsection{Modeling Module}

The modelling component allows for a simplified modelling of MST processes (sequential and parallel). Three major elements allow for the description of a complete production process.

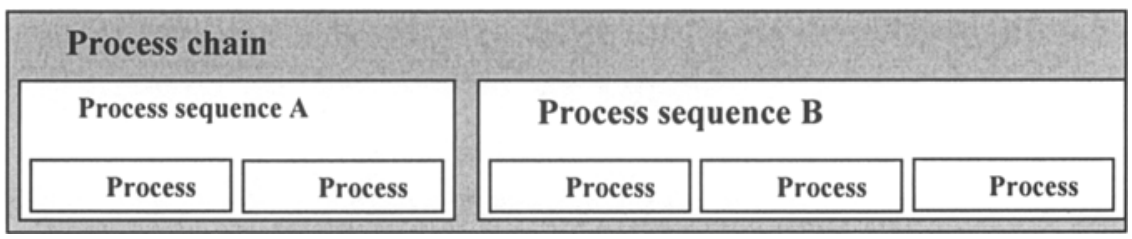

Fig. 6. Scheme of the major modelling elements in ProWiDa

The process chain element represents an order/product specific set of process sequences. It is equivalent to the model of a product or customer related production process. The process sequence represents a characteristic of a basic technology. The technology is represented by a set of process steps. The process step itself is the smallest modelling element, which represents a subtask that has to be executed when processing a technology. For further detailing the use of more sophisticated modelling methods like Petri Nets is more adequate [07]. 


\subsection{Interfaces for integration in Enterprise Resource Planning and other Business management systems}

In addition to the nearly "product independent" acquisition and storage of parameters, the ProWiDa system allows the collection of test and measurement data associated with of the iterative execution of a production or development process from feasibility studies up to the industrialisation. The data are stored in both ways, according to the product oriented work lists and as another view to the data pool in the way described above. Administrative features such as the definition of number ranges, batch tracing and statistical process control are also a part of this module.

For the manual acquisition of test and measurement data the ProWiDa System allows the automatic generation of process data sheets. The relevant process parameters for each process step can be derived from highlighted parameters of the equivalent competences in the parameter definition module. A "History" function supports the documentation of test series during the development phase.

Since the ProWiDa System is not intended to become an isolated tool in the enterprise software environment, connectors to ERP-systems had been defined. These connectors will allow for the exchange of accompanying information about process chains and their process steps in a technological and economical context (e.g. test and measurement data, process sheets and parts lists) to the ERP System and vice versa. Workflow functionality enables the model execution especially in the development phase, if there is no support by the ERP system. These functions are of extreme importance for user acceptance. For an easier integration the ProWiDa Integrating Infrastructure comparable to a service oriented architecture (SoA) approach had been developed allowing for an easier connectivity to other standard ERP software with XML interfaces (Fig 7).

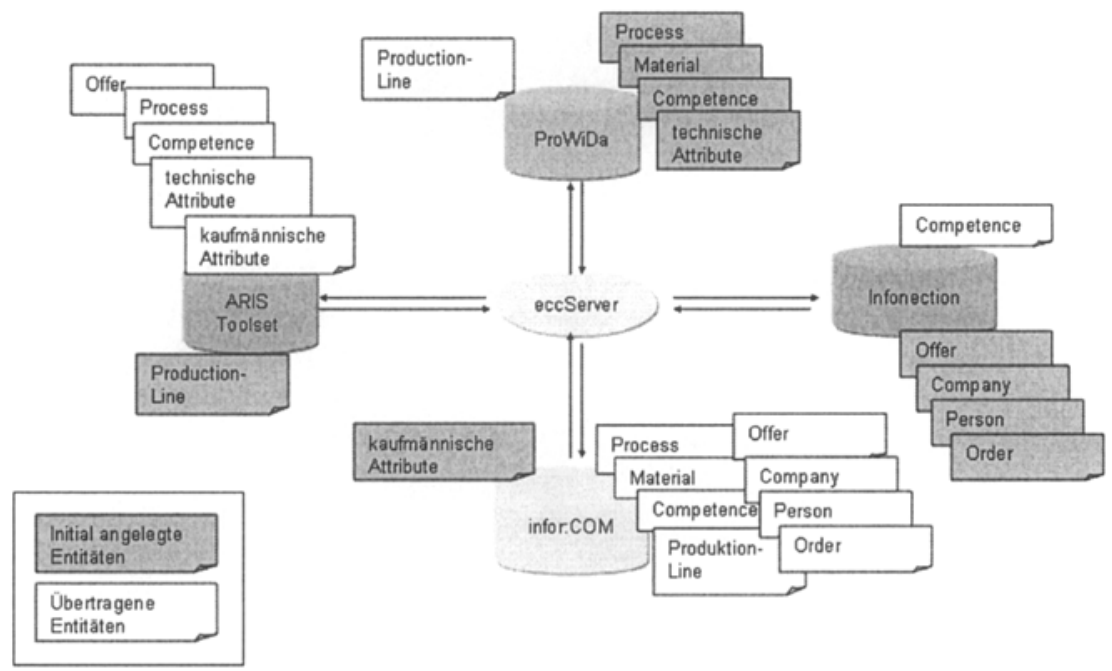

Fig. 7. The ProWiDa "Integrating Infrastructure" 


\subsection{Action planners as an approach for recursive detection of the "ideal" process chain}

Most tools available for process analysis and control focus on the aspects of "Total quality management" offering features like statistical process control etc., which are also covered by the batch tracing and test data module of the ProWiDa System.

In addition the ProWiDa System consists of a "process chain analyser". This prototype approach for detection of the most appropriate process chain bases on ideas coming originally from action planner approach This approach is quite common in the track planning of robots. The prototype approach was developed, to allow for an identification of potential solution paths, coming from a product requirement.

Pre-, runtime, and post- conditions had been defined for description of the "behaviour" of the competences. A software routine allows the comparison preconditions of a process step with post conditions of process steps stored in the central ProWiDa database [07].

The basis for this approach is the development of a MST description language that needs to be established and further developed. First ideas for a MST markup language, also coming from the field of Si-MST attest the feasibility of such an approach [08]. The ProWiDa process chain detection system in its current state demonstrates that such an approach, based on the modelling of MST process step behaviour, could be appropriate for future detection and benchmarking of MST technologies compared to a specific product requirement.

\section{Summary and Outlook}

The paper basically describes a knowledge management approach for MST production technologies allowing for a product independent definition, storage and retrieval of design rules, business rules and process parameters for the development of micro system products according to customers' needs. The approach is based on a generic database concept for the flexible combination of parameter sets attached to product and fabrication relevant aspects (e.g. technology, design, material, machines tools).

First prototype interfaces to enterprise information systems and components for analysis of data collected in this specific manner have been realised or are under development. They will allow for the import of measuring data from the production for a continuous optimisation of business rules/design rules based on "real world" process data. A very first prototype of a process chain analyser -allowing for an identification of the most appropriate solution path related to a specific task givenhas been developed and will be enhanced in the next few years. 


\section{References}

[01] Nonaka, I.; Takeuchi, H.: The Knowledge-Creating Company: How Japanes companies create the dynamics of innovation. Oxford University Press, New York; 1995.

[02] Heisig, P. in Report Wissensmanagement Hrsg.: C. H. Antoni, T. Sommerlatte, 4. Auflage 2001, Symposion Verlag

[03] Bouwstra, S., Da Silva, M.; Schroepfer, G.; White paper: Towards Standardization of MEMS Materials Characterization; Conventor Inc.; 2003

[04] Wagner, A. Hahn, K.; Considerations for MEMS physical design states, Sophia Antipolis Microelectronics Forum, France; 2003

[05] Brueck, R.; Dickerhof, M.; Hahn, K.; Langbein, I.; Towards an integrated design approach in Si+non Si MEMS Methodology; Presentation at the Patent-Dfmm Workshop in Berlin, Germany, 2007,

[06] Dickerhof, M.; Gengenbach, U.; Kooperationen flexibel und einfach gestalten, Hanser Verlag Munich, Germany, 2006 (only in German language)

[07] Dickerhof, M., Didic, M., Mampel, U.; Workflow and Cimosa -background and case study; Computers in Industry 40 P. 197-205, Elsevier Science, 1999

[08] Niedermaier, M., Entwicklung und prototypische Implementierung eines Konzepts zur Modellierung von prozessschritt-übergreifenden Aspekten der Mikrosystemtechnik auf der Basis von Technologieontologien; Diploma thesis; University of Karlsruhe, Faculty for Computer Science, Germany, 2007 (only in German language)

[09] Wagener, A.; Popp, J.; Hahn, K.; Brück, R.; PDML - A XML Based Process Description Language. In: Proceedings of the 9th European Concurrent Engineering Conference, Modena, 2002. - ECEC 2002 